\title{
LA RESPONSABILIDAD SOCIAL CORPORATIVA COMO PUNTO DE ENCUENTRO
}

\author{
Francisco Aguadero Fernández ${ }^{1}$ \\ Socio docente de DIRCOM \\ Aguadero@acta.es \\ www.acta.es/Aguadero
}

\begin{abstract}
Material autorizado para su primera publicación como artículo en la revista académica REDMARKA. Revista Digital de Marketing Aplicado.

https://doi.org/10.17979/redma.2010.01.05.4713
\end{abstract}

\section{RESUMEN}

La Responsabilidad Social Corporativa (RSC) es una disciplina en construcción y como tal viene cargada de grandes aportes y también de interrogantes a resolver. El presente artículo invita a la reflexión sobre alguno de esos interrogantes, analiza el estado de la disciplina, así como el papel de la terminología y el lenguaje del entorno de la RSC en el contexto de las organizaciones. Se propone el uso de una terminología normalizada en el interior de la empresa o institución como herramienta de gestión en las relaciones con los grupos de interés y con la sociedad, tendente a un mejor

\footnotetext{
1 Doctor en Filosofía y Ciencias de la Educación, Licenciado en Geografía e Historia, Diplomado en Ciencias Humanas, estudios complementarios en Marketing y Comunicación. Su tesis Doctoral versa sobre: "La Tecnología Educativa como un medio para la Comunicación y el desarrollo de la Organización", lo que le ha llevado a especializarse en Desarrollo, Comunicación, Cultura, Innovación y Responsabilidad Social Corporativa. Comunicólogo, escritor y periodista. Socio Docente de DIRCOM (Asociación de Directores de Comunicación); Miembro de ACTA (Asociación de Autores Científicos, Técnicos y Académicos) y de AIPET (Asociación Iberoamericana de Periodistas Especializados y Técnicos) Profesor principal en el Máster de RSE de la Universidad de Alcalá y codirector de la Maestría en Comunicación y Relaciones Públicas de la Universidad Autónoma de Guerrero (México). Consejero y asesor de varias entidades y revistas. Autor de dieciocho obras que son punto de referencia para profesores, empresarios, directivos, político, ejecutivos, profesionales, estudiantes y público en general. Ocupó varios puestos de alta responsabilidad en 3M, entre ellos el de Director de la Fundación. Conferenciante nacional e internacional.
} 
entendimiento y un funcionamiento más eficiente de cada empresa o institución concreta, así como para la sostenibilidad empresarial e institucional. La gestión del lenguaje es una parte importante para el óptimo aprovechamiento de los activos intangibles y del capital intelectual de toda organización.

La empresa debe evolucionar no hacia un cambio en la perspectiva y en el modelo de gestión, sino hacia un nuevo rol que pueda resultarle beneficioso para sí, para los grupos de interés y para la sociedad en general, debe tender a ser una "empresa ciudadana".

Pero está claro que tanto para la empresa como para las instituciones, la RSC es una tarea que hay que ir haciendo. No hay ni modelos estandarizados ni etapas o estadios puros. Lo importante es que cada una encuentre su camino, lo vaya haciendo y rinda cuentas a los grupos de interés y a la sociedad por medio de una comunicación transparente y responsable.

Palabras clave: Responsabilidad Social Corporativa, comunicación, sostenibilidad, terminología.

\section{ABSTRACT}

THE CORPORATE SOCIAL RESPONSIBILITY AS ENCOUNTER POINT

The Corporate Social Responsibility (CSR) is a discipline under construction and it comes with great contributions and also questions to be resolve. This article invites to reflect on any of these questions, analyzes the discipline's situation, as well as role of the terminology and language environment of CSR in the context of the organizations. It proposes the use of an standardized terminology inside the company or institution as a tool for managing relations with interest groups and society, that tries to find a better understanding and a for the smooth running of each company or specific institution, as well as for the corporate and institutional sustainability. The language's management is an important part for the optimal use of intangible assets and of any organization's 
intellectual capital. The company should not evolve a change in perspective and management model, but towards a new role that can be beneficial for themselves, for stakeholders and society in general, it should aim to be a "citizen enterprise".

But it is clear that for both of business and institutions, CSR is a path that it should be done step by step. There is no standardized models or pure stadiums to follow them in the same way. It is important that each finds its model, following it getting in touch with the stakeholders and society by means of a transparent and accountable communication.

Keywords: Corporate Social Responsibility, communication, sustainability, terminology. 


\section{Reflexiones sobre RSC}

Al acercarnos a lo que venimos llamando Responsabilidad Social Empresarial o Corporativa (RSE/RSC) nos surgen una serie de interrogantes en relación con la semántica. Las palabras, conceptos, terminología o lenguaje utilizado no son unívocos. Tienen muchas implicaciones y por tanto la polisemia es una constante.

A pesar de que llevamos unos años en el fragor del debate y de la práctica de la RSE continúan sin resolverse y sin consenso firme preguntas fundamentales como las siguientes, entre otras:

¿Se puede hablar de RSC/RSE con el tipo de economía en vigor?

¿Cabe la RSC sin antes interrogar y cuestionar el modelo de empresa actual?

¿Es la RSC un utilitarismo puro y duro por parte de las empresas?

¿Es simplemente una conveniencia de las empresas?

¿Será la RSC algo efímero?

¿Corre la RSC el riesgo de ser o convertirse en una simple cosmética?

¿Qué queremos decir cuando hablamos de RSC/RSE?

¿Podemos hablar de RSC si hay empresas que no asumen como finalidad hacer el bien?

¿Por qué se nos dice que una empresa no es una organización filantrópica o caritativa?

¿Es suficiente añadir la palabra ética para resolver cualquier problema?

¿No están las empresas instrumentalizando la ética para su interés particular?

¿Por qué nos centramos sólo en la responsabilidad de la empresa?

¿Cómo incide la responsabilidad individual en la empresarial o corporativa?

¿Qué incidencia tiene la responsabilidad empresarial en la sostenibilidad?

¿Debe ser voluntaria la RSC, o puede ser obligatoria?

Evidentemente, el grado de respuesta o asunción a estos interrogantes es muy variado según autores, empresas e instituciones, pero hemos de tener en 
cuenta que, hoy por hoy, en la economía imperante continuamos hablando de bancos, de empresas y de comercio, a pesar de que a las transacciones bancarias las llamemos éticas, las empresas éticas continúen persiguiendo el beneficio como última meta y el comercio justo siga basándose en acciones de compra-venta en cuyas negociaciones lo que se ventila es el beneficio.

\section{La función y el aporte de la terminología a la empresa}

No cabe ninguna duda de que el principal vector de relación entre la empresa y sus grupos de interés es la comunicación, sustanciada ésta en unos contenidos a transmitir y usando una determinada terminología. Resulta pues evidente y fácil de comprender la importancia que tiene el uso eficiente de la terminología en el contexto de las organizaciones y como vehículo para una adecuada comunicación en los procesos que se llevan a cabo, tanto dentro como fuera de la empresa o institución y en relación con los grupos de interés. En el interior de las organizaciones nos encontramos con una serie de actores cuyas relaciones se caracterizan por un uso terminológico específico. Dichos actores los podemos agrupar de la siguiente manera: directivos, ejecutivos, profesionales expertos monolingües, empleados bilingües, obreros 0 funcionarios y personal de apoyo. Por otra parte y desde el punto de vista de las funciones, en las organizaciones tenemos al personal financiero, informáticos, ingenieros, investigadores sociales o de mercado, recursos humanos, administrativos, operarios, u otros. Cada uno de estos grupos se expresa con un lenguaje y una terminología específica propia que los singulariza respecto de los otros.

Pensamos que, en aras de la eficacia, habría que llevar a cabo una adecuada sistematización de esas terminologías y construir un sistema de comunicación que sirva de guía en los procesos de intercambio de información entre los diversos grupos de interés de la empresa o institución. El instrumento sería la creación de una base de datos terminológica (BDT) que recoja toda la terminología utilizada en una empresa o institución en cuestión. Ello facilitará la comunicación entre los empleados o funcionarios y los procesos o actividades 
que se llevan a cabo, así como con el público exterior. Estamos con Heribert Picht (Picht, 1994: 91-97) cuando dice que "la empresa es uno de los productores y consumidores más importantes de terminologías... Así, la terminología es una herramienta en el conjunto del proceso productivo y debe considerarse como factor de producción. Producir y vender un producto sin documentación es impensable; la documentación forma parte integrante del producto; y en muchos sectores de servicio y consulta, la documentación misma representa el producto". Nos atrevemos pues a proponer el uso de una terminología normalizada o, si se quiere convenida, en el interior de la empresa o institución y para con los grupos de interés. Ello resulta de gran importancia para evitar que diferentes colectivos o grupos estén utilizando el mismo término pero para designar realidades distintas o muy diferenciadas entre sí.

La función y el aporte del uso correcto de la terminología en las organizaciones son un factor determinante para la productividad y la consecución de resultados positivos. Sin una terminología adecuada y normalizada no hay comunicación aplicada, profesional y eficaz, y, si ésta no se da, los diferentes flujos del proceso de interacción, del intercambio de ideas, compromisos y consensos necesarios para la realización de un buen trabajo y de una eficiencia empresarial o institucional acorde con los tiempos, tampoco se producirá.

Carolina Popp (Popp, 1994: 99-107) presenta una experiencia práctica en relación con lo tratado en este apartado y estamos con ella en que el uso de una terminología normalizada por medio de una base de datos terminológica significa un gran aporte para el fortalecimiento y desarrollo de cualquier tipo de organización, a nuestro entender, por la vía de:

- Mayor calidad de los servicios de auditoria y control.

- Respuesta más rápida en la preparación de informes para el cliente.

- Utilización más efectiva de las capacidades de los empleados o funcionarios.

- Ahorro de tiempo, debido a una mejora en la comunicación entre todos los implicados. 
- Y, como consecuencia, una mejora significativa de productividad.

Todo ello nos pone de manifiesto la importancia de usar una terminología normalizada para optimizar las actividades en las organizaciones y, por lo tanto, la rentabilidad y la satisfacción de los grupos de interés, así como la sostenibilidad institucional.

La forma en que tanto la información como el conocimiento se transfieren a los grupos de interés es una constante en la gestión empresarial o institucional para la consecución de una mayor efectividad y desarrollo de aquellas. La terminología se encuentra presente en la organización como instrumento fundamental que proporciona la claridad conceptual necesaria e indispensable para la transmisión, sin obstáculos, de los mensajes y buscando el rendimiento, prestigio y evolución de las organizaciones en cuestión. Dicho precepto lo encontramos en la comunicación aplicada, y para que ésta se dé en forma apropiada es preciso desterrar ambigüedades conceptuales e ir en busca de la armonía conceptual, puesta de manifiesto por medio de una terminología normalizada como herramienta de la gestión en las relaciones con los grupos de interés y con la sociedad.

\section{La gestión del lenguaje en la empresa o institución}

Aunque sin ánimo de agotar toda la polisemia habida en torno al lenguaje, sí resulta indispensable acercarnos a una definición de lo que representa el uso de aquél como elemento clave que estimula las relaciones con los grupos de interés y el desarrollo de las organizaciones.

Los nuevos retos y tendencias que el mundo globalizado de hoy plantea, requieren de la creación, utilización y aprovechamiento del talento, así como de nuevos recursos lingüísticos para el desarrollo empresarial, económico y social. Porque la sociedad de la información y del conocimiento lleva en sus genes el compartir los conocimientos de las personas al objeto de crear valor y generar 
innovación, encontrando ambas la expresión más viva en la disposición de productos y servicios que nos posibiliten una vida mejor y más fácil.

Para nosotros y a estos efectos, contemplamos la gestión del lenguaje como una parte importante para el óptimo aprovechamiento de los activos intangibles y del capital intelectual de toda organización. Entendiendo éste último a la luz de las aportaciones de Brooking (Brooking, 19973: 250) como el conjunto de activos inmateriales que hacen posible el funcionamiento de la empresa.

\section{Conceptualización y etapas de la RSC}

En una disciplina finalista como es la RSC no somos partidarios de precisar tempranamente su denominación ni su alcance. Entre otras cosas, porque la práctica empresarial e institucional no se para, ella va haciendo y espera que los términos conceptuales se los de resueltos la academia, la universidad. Así ha ocurrido con la RSC, la empresa se ha puesto en marcha y va haciendo con sus estrategias y programas al respecto. Pero a diferencia de otras ocasiones 0 disciplinas, en el caso de la RSC la empresa si se ha lanzado desde los comienzos a aportar sus propias definiciones de la RSC, de tal suerte que el número de definiciones que podemos encontrarnos para denominar a eso que conocemos como del ámbito de la RSC es altísimo. No hay un consenso ni una cifra más creíble que otra, pero se escucha hablar de treinta, más de cincuenta o setenta y tantas definiciones, para todos los gustos. Si bien, son los conceptos de RSC o RSE (Responsabilidad Social Empresarial) los que hasta el momento son más utilizados.

A la espera de que el análisis y debate que se está llevando a cabo en lo académico llegue a un consenso y sin desmerecer a toda esa variedad de definiciones, traeremos aquí la de este autor y las de las instituciones más representativas. Así, en 1993 Aguadero (Aguadero, 1993: 161) la definía como "la capacidad de compromiso que toda persona u organización asume para responder ante la sociedad de las consecuencias derivadas de sus actos". 
Por su parte, la Comisión Europea (2001) definía a la RSE como "la integración voluntaria, por parte de las empresas, de las preocupaciones sociales y medioambientales en sus operaciones comerciales y sus relaciones con sus interlocutores". La AECA (Asociación Española de Contabilidad y Administración, 2003) definía a la RSE como "el compromiso voluntario de las empresas con el desarrollo de la sociedad y la preservación del medio ambiente, desde su composición social y un comportamiento responsable hacia las personas y grupos sociales con quienes se interactúa". De otro lado, el Ministerio de Trabajo y Asuntos Sociales (2005) tras hacerse eco de las consideraciones de diferentes organizaciones, definía a la RSE como "un conjunto de compromisos de diverso orden económico, social y medioambiental adoptados por las empresas, las organizaciones e instituciones públicas y privadas y que constituyen un valor añadido al cumplimiento de sus obligaciones legales, contribuyendo a la vez, al progreso social y económico en el marco de un desarrollo sostenible". Por su parte, la red Forum Empresa (www.empresa.org/que_es_la_RSE) que es la organización más extensa del continente americano en temas de RSE, entiende la Responsabilidad Social Empresarial como "una nueva forma de hacer negocios, en la que la empresa gestiona sus operaciones en forma sostenible en lo económico, social y ambiental, reconociendo los intereses de distintos públicos con los que se relaciona, como los accionistas, los empleados, la comunidad, los proveedores, los clientes, considerando el medio ambiente y el desarrollo sostenible de las generaciones futuras".

Recientemente se ha comenzado a hablar de la RSE 2.0 con distintos significados. Para unos significa la utilización de las herramientas que posibilita la web 2.0 desde el punto de vista de comunicación y de relaciones; y para otros significa el incorporar la RSE al núcleo central, al ADN de la empresa o institución en cuestión.

Definiciones éstas de la RSC/RSE pioneras, representativas y sucesivas en el tiempo (1993, 2001, 2003, 2005, 2007 y 2009) y en la representación geográfica: España, Europa, América; así como de distintos colectivos e 
instituciones: experto, asociaciones profesionales, Ministerio, Comisión Europea. Pero, que como decíamos más arriba, hay otras muchas definiciones tan válidas como estas.

Aún a riesgo de simplificar y sin perjuicio de lo que digan otras voces autorizadas, podemos decir que la RSC llegó a las empresas promovida por la función de comunicación, no en vano, en una gran parte de las empresas la RSC está vinculada a los departamentos de comunicación hasta tanto se hace mayor y se independiza y, lógicamente, en esa etapa la RSC se centra, en gran parte, en contar lo que al respecto hace la empresa. Luego viene una segunda etapa en la que la RSE pasa a formar parte de la estrategia de la empresa, más vinculada al negocio central de la misma; y una tercera en la que la RSE se incorpora a la cuenta de resultados siendo el motor para la localización y generación de nuevos mercados.

Otra perspectiva de análisis de la implantación y evolución de la RSE es aquella que, en primer lugar, ve a la RSE como una demanda más del mercado, aunque más compleja, y por ahí se cuelan los conceptos de "Marketing social" y similares. Luego se ve a la RSE como un paraguas para los nuevos riesgos que trae la sociedad de la información y que pudieran afectar a la reputación de la empresa. Una empresa bien posicionada en RSE crece en reputación y se hace más fuerte ante cualquier circunstancia negativa. El tercer estadio desde esta perspectiva consistiría en ver a la RSE como una nueva área de gestión a consolidar dentro del organigrama de la empresa. Las acciones más visibles de este estadio de la RSE son las contribuciones económicas y en especies a las causas sociales y también por ahí la terminología se hace patente en conceptos como el "Marketing con causa".

Cualquiera de los estadios analizados anteriormente y desde cualquiera de las dos perspectivas tratadas, son compatibles y pueden darse en una empresa dotada de una misión, una visión y unos valores determinados. Ahora bien, la RSC/RSE pasa a un estadio superior cuando la empresa se dota de la vida moral que aquellos valores le proporciona, para asentar sobre los mismos las 
relaciones con los grupos de interés y con la sociedad, con una visión estratégica en la que los grupos de interés y la sociedad son el eje central de su sostenibilidad y su desarrollo.

Comparto con Itziar Castelló y José M. Lozano del Instituto de Innovación Social de ESADE (2009) la posible existencia de una cuarta etapa en la que encontraríamos a la denominada "empresa ciudadana" etapa ésta en la que la RSE estaría formando parte de la propia misión de la empresa, es decir, determinando la propia "razón de ser" de la empresa en cuestión. En este estadio la empresa se plantea en que tipo de sociedad quiere vivir y como puede contribuir a ello. Con esto no nos estamos refiriendo a un cambio en la perspectiva y en el modelo de gestión, sino en un nuevo rol de la empresa que pueda resultar beneficioso para si, para los grupos de interés y para la sociedad en general. Puede que todavía no haya ninguna entidad merecedora de este carácter de "empresa ciudadana" pero si existen empresas con iniciativas y programas en ese sentido. La compañía 3M, por ejemplo, desde hace unos cuantos años lleva entre sus comportamientos éticos y de responsabilidad lo de ser un "buen ciudadano", algo exigido dentro de la Compañía y proclamado fuera. Así, desde los años veinte del siglo pasado, esta entidad comenzó a preocuparse por los aspectos de salud, seguridad e higiene de sus empleados y de la sociedad, y en los años setenta inició programas medioambientales con el convencimiento de que la prevención de la contaminación es rentable.

A estas alturas parece claro que tanto para la empresa como para las instituciones, la RSC es un camino que hay que ir haciendo. No hay ni modelos estandarizados ni etapas o estadios puros. Lo importante es que cada entidad encuentre su camino y lo vaya haciendo.

\section{EI lenguaje de la RSE en las pequeñas y medianas empresas (PYMES)}

Tanto en la grande como en la pequeña y mediana empresa hemos de observar el lenguaje de la RSE desde una doble aproximación que resulta a la 
vez social y utilitarista. Este enfoque se hace más necesario en la PYME que puede no se sienta tan identificada como las grandes empresas con determinadas expresiones o vocablos, aún cuando, como éstas, persiga un interés pragmático junto con otro orientado a lo social.

Es preciso que la PYME no perciba el lenguaje de RSE como un leguaje normativo e impuestos que la presiona. Será mejor y más eficaz si lo percibe como una oportunidad para una mejora de su gestión y para una mayor integración con el entorno que la rodea.

Frente al gran concepto de Responsabilidad Social Corporativa, para las PYMES puede tener más sentido el de Gestión Responsable, recogiendo con el vocablo Gestión el aspecto pragmático, utilitarista y de competitividad que necesariamente ha de tener la PYME; y con el vocablo Responsable atender a la responsabilidad derivada de sus relaciones con los agentes necesarios para su supervivencia y aporte a la sociedad.

Pensamos que conceptos más próximos y habituales como hacerlo bien, buen trato, calidad, responsabilidad, compromiso, iniciativa, proactividad, emprendedora, competitividad responsable o confianza, llegan mejor a la PYME y son más efectivos que otros más abstractos y centrados en lo ético, en lo social e incluso en los valores.

Esta conveniencia de adaptación del lenguaje para las PYMES está en línea con la postura expresada por parte de alguna de las organizaciones empresariales en el sentido de hablar de la Responsabilidad Social de la PYME (RSP) para poner de manifiesto la especificidad de éstas en un asunto tan importante como transversal.

\section{Contenido y estructura de la comunicación sobre RSC}


El conjunto de elementos que intervienen en la comunicación de la RSE es extenso y variado. Ahora bien, de cara a la realización de este trabajo hacemos dos grandes apartados: uno que contempla los aspectos terminológicos, de lenguaje, anglicismos, semántica y mensajes utilizados para expresar lo referido a la RSC/RSE y otro, referido al contenido de la información sobre responsabilidad social de la empresa o institución. Nos hemos venido ocupando del primer apartado y en este capítulo haremos un acercamiento al segundo.

Desde los inicios del siglo $X X I$, pocos años a, comenzaron a publicarse informes y memorias empresariales de RSC y de Sostenibilidad, siendo las grandes empresas las principales generadoras de las mismas. Enseguida se cuestionaron dos aspectos fundamentales de la comunicación: lo referido a los contenidos en cuanto a qué información se debe facilitar; y lo concerniente a la estructura y la forma de presentarla.

Consecuentemente, surge la necesidad de una normalización que generalmente ha sido aceptada por diversos factores y entre ellos:

1. La conveniencia de establecer unos criterios comunes entre organizaciones, grupos de interés y verificadores de la información que se hace pública.

2. Proponer elementos de referencia para la verificación por independientes, incrementando así la credibilidad de la información.

3. Orientar a las empresas e instituciones sobre la información más relevante a publicar y cómo estructurarla.

4. Posibilitar la comparación entre las organizaciones que informan.

5. Aprender unas organizaciones de otras.

6. Identificar las mejores prácticas.

7. Proporcionar a los consumidores y a los diferentes grupos de interés instrumentos de referencia. 
Todo lo anterior ha sido posible gracias a La Global Reporting Initiative (GRI) que ha elaborado y proporcionado un modelo sistémico de información para comunicar sobre RSC y que está estructurado teniendo en cuenta las tres dimensiones del concepto de sostenibilidad: la económica, la social y la medioambiental. Este modelo tuvo buena acogida inicialmente por parte de las organizaciones y se está asentando como referente y en la aplicación de las sucesivas versiones. No obstante, esta guía no pretende, ni puede ser un formato único e inamovible como el patrón de la contabilidad financiera para las cuentas anuales de resultados. La naturaleza de la información que alberga requiere de una flexibilidad que permita recoger y destacar los aspectos propios y más singulares de cada organización. Por ello, la propuesta del GRI para la estructura de presentación de la información es simplemente orientadora, puesto que cada memoria o informe ha de contemplar las características peculiares de la empresa o institución en cuestión.

Observamos pues que el contenido y el formato de presentación de la información sobre RSC varían de una empresa a otra y a veces sustancialmente, con las consecuentes complicaciones para la comparación entre empresas en el ejercicio y la puesta en práctica de sus respectivas responsabilidades corporativas. El modelo sistémico del GRI viene a acotar esa dispersión haciendo posible la comparación mediante una estandarización generalmente aceptada y compartida para un tratamiento de la información económica, social y medioambiental. La propuesta del GRI concibe una memoria de sostenibilidad estructurada en los siguientes apartados:

1. Visión y estrategia empresarial, respecto a la sostenibilidad

2. Perfil de la organización que está informando

3. Parámetros de la Memoria en cuestión

4. Gobierno y sistemas de gestión de esa entidad (compromisos, participación $\mathrm{Gl}$ )

5. Indicadores de actuación económica, medioambiental y social (con un índice de los mismos si estuvieran dispersos)

6. Autocalificación (del nivel de aplicación) 
Pero como decíamos más arriba, la guía que aporta el GRI no pretende, ni puede ser un formato único e inamovible de comunicación de la RSC, de hecho, empresas como Telefónica, avanzada en temas de RSC y pionera en la aplicación de aquella metodología, en su Informe Anual de Responsabilidad Corporativa del 2009 cambia la manera de rendir cuentas ante los grupos de interés y de la sociedad y lo hace basándose en los 10 principios del Pacto Mundial de Naciones Unidas, pretendiendo así caminar hacia un modelo común y comparable entre entidades de diverso índole.

\section{Aplicación terminológica a una campaña institucional}

Para una mejor expresión y entendimiento trataremos de ilustrar las ideas de este apartado tomando como ejemplo el caso de Iberdrola. Nos parece significativa la Campaña institucional de Iberdrola (http://www.iberdrola.es) que perseguía incrementar los valores de la Compañía y fortalecer su imagen de marca. Estratégicamente se aprovechó la participación en el deporte de vela con el "Desafío" español asociando los siguientes valores temáticos:

- Desafío

- La fuerza

- La calidad

- La naturaleza

- La expansión

- La innovación

- $\quad$ El equipo

- $\quad$ La rentabilidad

Para terminar diciendo: "queremos ser tu energía" consiguiendo así el máximo acercamiento al cliente, al consumidor. 


\section{Crisis económica, RSC y futuro}

Un somero análisis de los acontecimientos de los últimos años pone de manifiesto las distintas etapas de la crisis global que nos llevó a la gran recesión mundial y que pudimos constatar en 2009. Se inició con una crisis alimenticia debido a los altos precios de alimentos básicos como los cereales y que afectó, sobretodo, al tercer mundo. A ello le siguió la crisis de los combustibles con los precios del petróleo a cotas disparatadas. Seguidamente vino la crisis financiera que desembocó en 2008 en una crisis económica mundial de gran intensidad, con una pérdida de credibilidad en el sistema que afectó profundamente a la reputación de las empresas y de las instituciones.

Y ¿cuál ha sido el papel de la RSC en todo éste proceso? En las crisis anteriores podríamos decir que se habían producido por la ausencia de una responsabilidad por parte de los agentes implicados, pero, en esta ocasión, la RSC ya estaba presente en las grandes corporaciones con sus códigos de ética y de buen gobierno, entonces ¿qué fue lo que pasó?, ¿qué la RSC no sirve para nada? Lo que ha pasado, a nuestro entender, es que la RSC había llegado a las empresas y a las instituciones, pero no al corazón de las mismas. Se había quedado en la periferia, sin penetrar en el núcleo central, sin formar parte del ADN de las organizaciones, sin ser asumida y puesta en práctica, de verdad, por los directivos de aquellas. Con unas empresas basadas en la RSC, la crisis económica y posterior recesión del 2009 no se habrían producido.

Todo parece indicar que en tiempos de crisis, la RSC es un componente transversal de la organización que aporta coherencia a los planes, a los procesos de gestión y a las acciones, siendo determinante para que ganen en reputación aquellas empresas que estén bien posicionadas en RSE y que actúen de una forma socialmente responsable.

Por consiguiente, la reflexión no puede llevarnos a obviar la RSC/RSE porque estaba allí y no resolvió nada, sino todo lo contrario, debe potenciarse la misma 
y hacer que se incorpore a la propia razón de ser de las empresas e instituciones, obteniendo de esta manera empresas y directivos más responsables. Ello nos llevará a un sistema económico-social más justo y equilibrado. Ello nos proporcionaría una empresa más ciudadana.

Pero la crisis también nos pide la reflexión sobre la voluntariedad o no de la RSC. Todo parece indicar que volvemos a ese debate que ya parecía superado y del cual había salido un cierto consenso en que aquella debía ser voluntaria para las empresas. Después de lo que ha pasado con la crisis global y la gran recesión mundial ¿seguimos pensando en que eso es lo mejor? Algunos países ya han comenzado a legislar al respecto. Y, lo que sí es seguro es que la RSE será cada vez menos opcional para las empresas y para las instituciones porque la sociedad, los ciudadanos, los clientes o consumidores ya "piensan en verde" en un $95 \%$ a la hora de decidir su compra; el $54 \%$ de los consumidores valoran la sostenibilidad y el respeto al medio ambiente a la hora de evaluar un producto y pensar en comprarlo; y el $22 \%$ ya compra productos respetuosos con el medio ambiente (Según un estudio realizado por Deloitte en colaboración con la organización Grocery Manufacturers Associations).

Por otra parte, puede que también nos tengamos que ir acostumbrando a utilizar más el concepto de ciudadano en cuanto que persona responsable capaz de decidir, que el concepto de consumidor que consume lo que se le ofrezca y que tuvo su auge en otros tiempos.

\section{Consideraciones finales}

La evolución de la ciencia de la organización así como la práctica de la actividad organizativa ha traído sucesivos e importantes cambios pero en pocas ocasiones han afectado tanto al conjunto de la empresa y de las instituciones como lo está haciendo la implantación de la RSC, que se constituye como un punto de encuentro de todos los elementos que la 
componen: personal, capital intelectual, procesos, comportamientos, lenguaje, terminología, entorno, intereses, compromisos o capital social, entre otros.

Sostenibilidad, responsabilidad y transparencia son palabras claves para después de la crisis global, de la gran recesión de 2009 y para el futuro.

\section{Referencias bibliográficas}

Aguadero, F. (1993) Comunicación Social Integrada. Un reto para la organización. Ciencia 3, Madrid

Aguadero, F. (2003) La Sociedad de la Información. Vivir en el siglo XXI. Acento Editorial, Madrid

Aguadero, F. (2009) Innovación y Sostenibilidad. Ciencia 3, Madrid

AECA (2003) Marco Conceptual de la RSE, AECA, Madrid

Archel, P. y Larrinaga, C. (2005) Limites de la información de sostenibilidad: entidad, devengo y materialidad. AECA. Madrid

Brooking, A. (1997) El capital intelectual. Principal activo de las empresas del tercer milenio, Paidós, Barcelona

Castelló, I. y Lozano J. M. (2009) De la gestión del riesgo a la empresa ciudadana: Ios estadios de evolución de la RSE, ESADE, Barcelona

Comisión Europea (2001) Libro verde. Fomento marco europeo para la RSE, Bruselas

Lizcano, J. L. y Nieto, P. (2006) La Semántica de la Responsabilidad Social Corporativa. AECA, Madrid

Lizcano, J. L. y Moneva, J. M. (2004) Marco conceptual de la responsabilidad social corporativa. AECA. Madrid

Ministerio de Trabajo y Asuntos Sociales (2005) La RSE. Diálogo social, Madrid

Picht, H. (1994) "Terminología para la empresa y la industria" III Simposio Iberoamericano de Terminología, Riterm: Terminología y Desarrollo, Unión Latina, Buenos Aires

Popp, C. (1994) "Bancos de datos terminológico para la industria petrolera: una experiencia argentina" III simposio Iberoamericano de Terminología, Riterm: Terminología y Desarrollo, Unión Latina, Buenos Aires 
Fuentes para consulta

http://www.elportaldelaconciliacion.com

http://www.nexos.es

http://www.aeca.es

http://www.empresa.org

http://www.iberdrola.es

Para citar este artículo:

Aguadero Fernández, Francisco (17-12-2010). LA RESPONSABILIDAD SOCIAL CORPORATIVA COMO PUNTO DE ENCUENTRO.

REDMARKA - CIECID - Unidad de Investigación en Marketing Aplicado-Universidad de A Coruña

Año II, Número 5, V1, pp.53-70

ISSN 1852-2300

URL del Documento : cienciared.com.ar/ra/doc.php?n=1413

URL de la Revista : cienciared.com.ar/ra/revista. php?wid=39 